CLINICAL CASE

\title{
THYROID ULTRASOUND PERFORMED FIRST HAND BY THE ENT SURGEON
}

\author{
Daniela Vrînceanu ${ }^{1}$, M. Dumitru ${ }^{1,2}$, A. Costache ${ }^{3}$, R. Cergan ${ }^{2}$ \\ ${ }^{1}$ ENT Department, Bucharest Emergency University Hospital, Bucharest, Romania \\ ${ }^{2}$ Anatomy Department, "Carol Davila" University of Medicine and Pharmacy, Bucharest, Romania \\ ${ }^{3}$ Ultrasound Teaching Center, "Carol Davila" University of Medicine and Pharmacy, Bucharest, Romania \\ Corresponding author: Mihai Dumitru \\ Email: orldumitrumihai@yahoo.com
}

\begin{abstract}
The next level in thyroid disease management is the use of first hand surgeon performed sonography. Given the fact that many of the cases with thyroid carcinoma are referred to Ear Nose and Throat (ENT) departments, this diagnostic imaging modality should be available on site. We present a case series with thyroid pathology that benefited from sonography performed first hand by the ENT surgeon in a tertiary teaching hospital. Thyroid pathology is varied from benign cysts to papillary and medullar carcinomas. Further data are necessary for establishing the learning curve for this exam used by ENT surgeons. The foreseeable advantages are faster protocol in oncology cases, cost efficiency and wide scale availability of thyroid ultrasonography.
\end{abstract}

KEYWORDS: thyroid pathology, thyroid ultrasound, ENT surgeon

\section{INTRODUCTION}

Thyroid pathology presents an increasing trend in prevalence and incidence worldwide. Sonography represents the first diagnostic imaging tool in the differential diagnosis of thyroid masses. Unfortunately, the pathology of the thyroid gland ranges from benign autoimmune thyroiditis to undifferentiated anaplastic carcinomas. Facing these problems healthcare systems must improve services and expedite diagnosis and treatment. One possible solution to these menacing aspects could be the implementation of thyroid ultrasonography performed first hand by the Ear, Nose and Throat (ENT) surgeon. Backing this endeavor is the fact that actually many of the cases with thyroid masses are submitted to surgery in ENT and Head and Neck Surgery (HNS) departments and thus reduce the time lost with acquiring imaging studies in other departments [1]-[5].

\section{CASE PRESENTATION}

\section{Ultrasound protocol}

The ENT surgeon possesses in-depth knowledge of thyroid gland anatomy and neighboring structures. A complete ultrasound exam of the thyroid gland must follow the steps: 1) measurements of at least 2 diameters in 2 different planes of the thyroid lobes; 2) measurement of thyroid isthmus thickness; 3) documenting the possible pathology using classic ultrasound criteria - echogenicity, contour, calcifications, etc.; 4) imaging assessment of neck lymph nodes; 5) Doppler signal assessment plus elastography analysis when available. The average ultrasound learning curve for ENT surgeons is reduced to 30 days and a minimum of 50 thyroid ultrasound exams.

\section{Clinical Case Series}

We present ultrasound imaging findings in cases with thyroid pathology submitted to 
ENT Department at Bucharest University Emergency Hospital. We used a portable bedside Sonoscape S2 ultrasound machine equipped with a linear probe with variable working frequency. The most frequent pathology encountered is thyroid cysts (Figure 1). These could be true benign cysts or appear during the evolution of colloid deposits, hemorrhage or necrotic transformation of nodules [6], [7].

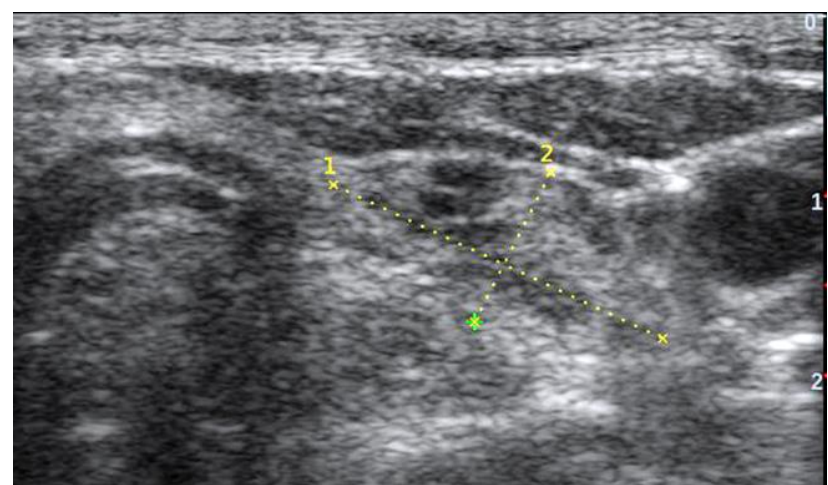

Figure 1 - Thyroid cysts developing inside a nodule

Another interesting finding is calcifications inside the thyroid parenchyma (Figure 2). These usually evolve in long lasting solitary nodules and are identified with the help of the ultrasound back shadow effect [8], [9].

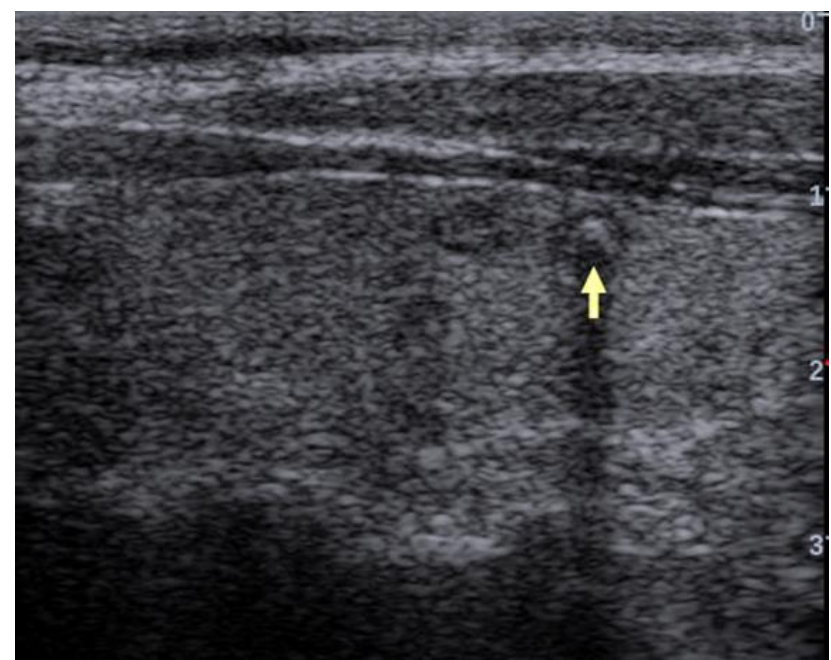

Figure 2 - Thyroid calcifications - arrow shows the back-shadow effect

The nodules (Figure 3) are more likely to be benign if they meet the following criteria: mostly cystic in nature, presence of comet tail effect, hyperechoic nodules, oval in shape, sharp hypoechoic contour, Doppler signal mostly in the periphery [10], [11].

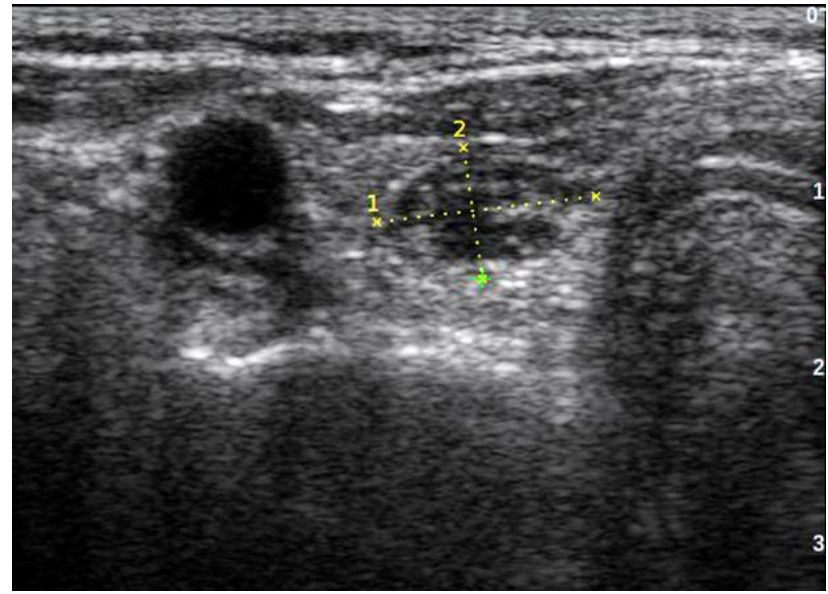

Figure 3 - Benign nodule

For a TIRADS 5 (Thyroid imaging reporting and data system) nodule (Figure 4) with more than $80 \%$ chance of malignancy should be considered the following aspects: hypoechoic structure, uneven borders, micro calcifications, rounder than oval in shape, Doppler signal inside the nodule [12], [13].

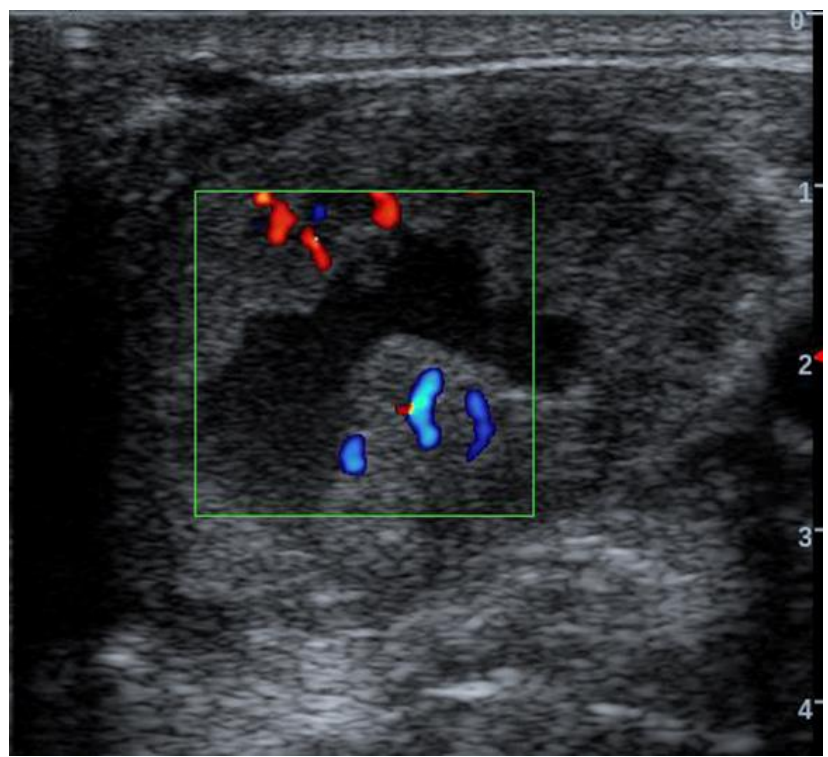

Figure 4 - Thyroid carcinoma - neoformation vessels inside the tumor

In cases with pathology affecting the isthmus (Figure 5) the most probable course of treatment implies total thyroidectomy [14]-[16]. 


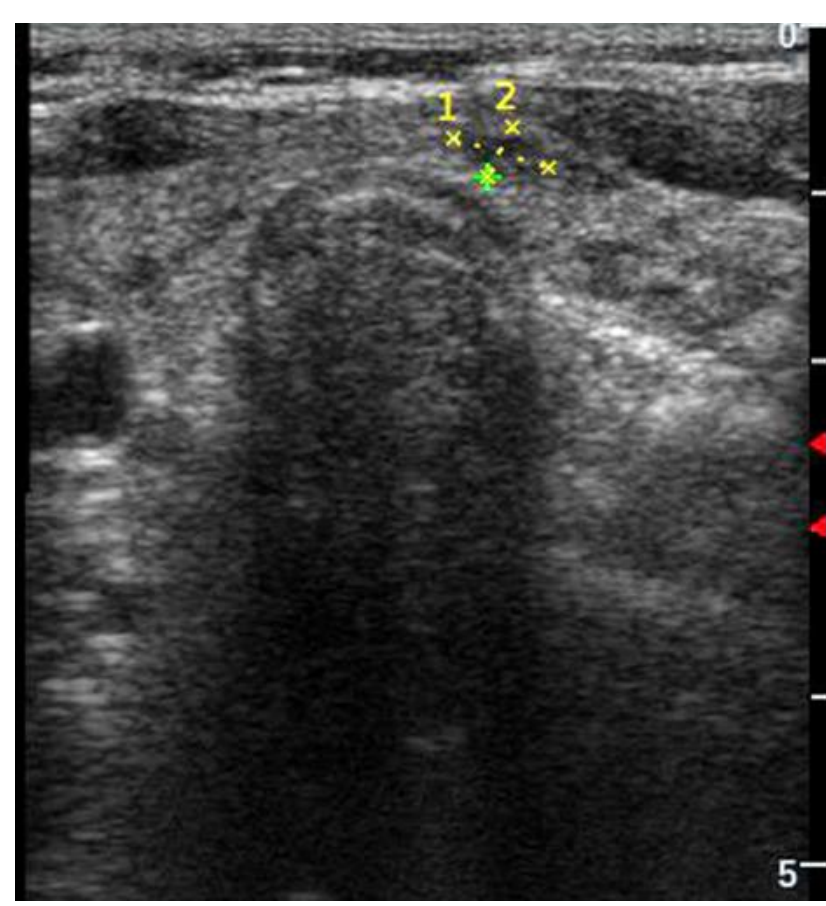

Figure 5 - Cyst at the level of the isthmus in a goiter patient

Another detail is the mass effect (Figure 6 ) of giant thyroid goiters over the main arteriovenous bundle of the neck along with possible lymph nodes involvement [17].

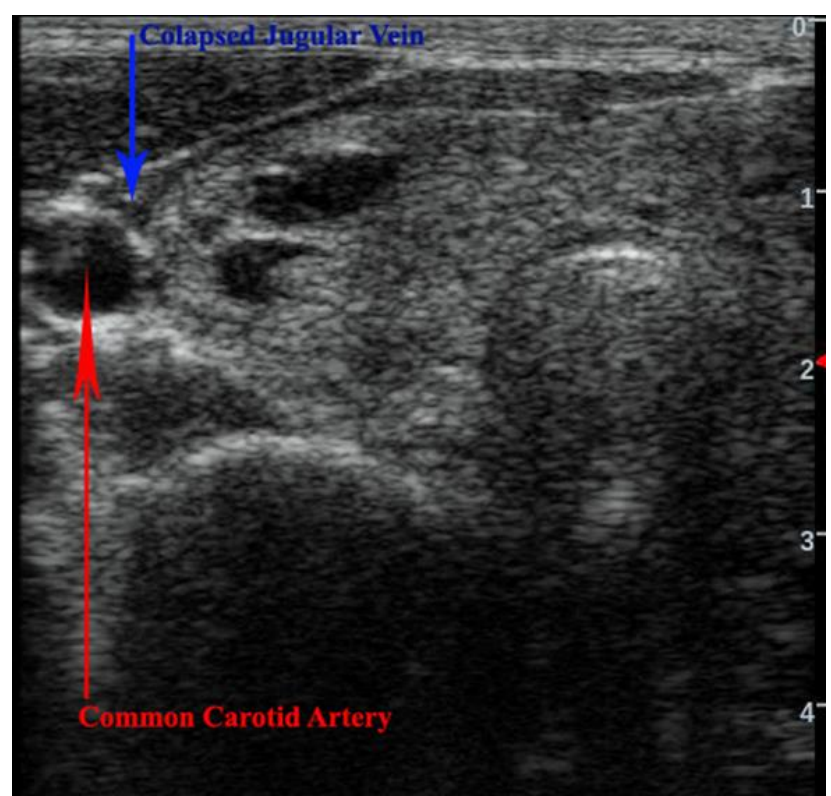

Figure 6 - Displacement of the carotid and jugular vessels at thyroid level

\section{DISCUSSIONS}

Ultrasonography has some clear advantages: fast procedure, lack of irradiation and wide scale availability nowadays. Thyroid sonography presents sensibility and specificity higher than $90 \%$ for differentiating benign from malignant pathology. This may be achieved by combining sonography criteria with the pathology results obtained through ultrasound guided fine needle aspiration biopsy. Current training practices in sonography credit ultrasound at the level of the thyroid gland with a reduced learning curve of more than 50 procedures in about 1 month of training [18].

\section{CONCLUSIONS}

Thyroid ultrasound performed first hand by the ENT surgeon could be the solution for the increasing number of cases with tumors requiring a fast diagnosis and treatment. Therefore, the management of the patient will be quicker without waiting lists in already crowded imaging departments. Nonetheless the patients' compliance to diagnosis steps and treatment should be increased. Also, the surgeon can directly analyze the extent of the pathology and plan dissection of neighboring anatomy structures.

\section{REFERENCES}

[1] A Mohebati, AR Shaha. Anatomy of thyroid and parathyroid glands and neurovasuclature relations. Clinical Anatomy. 2012; 25:19-31.

[2] A Costache, M Dumitru, I Anghel, R Cergan, AG Anghel, C Sarafoleanu. Ultrasonographic anatomy of head and neck - a pictorial for the ENT specialist. Med Ultrason. 2015; 17(1):104-108.

[3] JH Shin, JH Baek, J Chung, et al. Ultrasonography diagnosis and image-based management of thyroid nodules: revised Korean Society of Thyroid Radiology consensus statement and recommendations. Korean J Radiol. 2016; 17(3):370-95.

[4] D Vrinceanu, M Dumitru, R Cergan, AG Anghel, ET Patrascu, CC Sarafoleanu, A Costache. Correlations between ultrasonography performed by the ENT specialist and pathologic findings in the management of three cases with thyroglossal duct cyst. Med Ultrason. 2018; 0:1-3. DOI: 10.11152/mu1422.

[5] HS Hong, EH Lee, SH Jeong, et al. Ultrasonography of various thyroid diseases in children and adolecents: a pictorial essay. Korean J Radiol. 2015; 16(2):419-29.

[6] B Ma, Y Jia, Q Wang, X Li. Ultrasound of primary thyroid non-Hodgkin's lymphoma. Clin Imaging. 2014; 38(5):621-6 
[7] RA Sofferman, AT Ahuja. Ultrasound of the Thyroid and Parathyroid Glands. Springer. New York. 2012. pp61-106.

[8] T Fukuhara, E Matsuda, Y Endo, et al. Impact of fibrotic tissue on shear wave velocity in thyroid: an ex vivo study with fresh thyroid specimens. BioMed Research International. 2015; 2015:569367.

[9] H Malhi, MD Beland, SY Cen, et al. Echogenic foci in thyroid nodules: significance of posterior acoustic artifacts. AJR. 2014; 203(6):13106.

[10] S Sheth. Role of ultrasonography in thyroid disease. Otolaryngol Clin North Am. 2010;43(2):239-55

[11] JP Brito, MR Gionfriddo, A Al Nofal, et al. The accuracy of thyroid nodule ultrasound to predict thyroid cancer: systematic review and meta-analysis. J Clin Endocrinol Metab. 2014; 99(4):1253-63.

[12] LR Remonti, CK Kramer, CB Leitão, LC Pinto, JL Gross. Thyroid ultrasound features and risk of carcinoma: a systematic review and meta-analysis of observational studies. Thyroid. 2015; 25(5):538-50 [13] F Pagni, M Jaconi, A Delitala, et al. Incidental papillary thyroid carcinoma: diagnostic findings in a series of 287 carcinomas. Endocr Pathol. 2014; 25(3):288-96

[14] SH Lee, JS Baek, JY Lee, Predictive factors of malignancy in thyroid nodules with a cytological diagnosis of follicular neoplasm. Endocr Pathol. 2013; 24(4):177-83

[15] SY Hahn, BK Han, EY Ko, JH Shin, ES Ko. Ultrasound findings of papillary thyroid carcinoma originating in the isthmus: comparison with lobeoriginating papillary thyroid carcinoma. AJR Am J Roentgenol. 2014; 203(3):637-42

[16] YJ Kim, HS Hong, SH Jeong, EH Lee, JJ Kwak. Papillary Thyroid Carcinoma Arising Within a Follicular Adenoma: A Case Report, UltrasoundFeatures, and Considerations. Ultrasound Q. 2016 Apr 23

[17] AK Ganti. Anaplastic Thyroid Carcinoma: Light at the End of the Tunnel? J Oncol Pract. 2016; 12(6):519-20.

[18] IR Paunovic, SB Sipetic, GV Zoric, et al. Survival and prognostic factors of anaplastic thyroid carcinoma. Acta Chir Belg. 2015; 115:62-7. 